\title{
Delivering insecticide-treated nets for malaria prevention: innovative strategies
}

This article was published in the following Dove Press journal:

Research and Reports in Tropical Medicine

22 September 2016

Number of times this article has been viewed

\author{
Paul J Krezanoski ${ }^{1-3}$ \\ 'Department of Medicine, \\ 2Department of Pediatrics, \\ Massachusetts General Hospital, \\ ${ }^{3}$ Medicine and Pedatrics, Harvard \\ Medical School, Boston, MA, USA
}

Correspondence: Paul J Krezanoski Department of Medicine, Massachusetts General Hospital, 55 Fruit Street, Boston, MA 02II4, USA

Email pkrezanoski@mgh.harvard.edu

\begin{abstract}
The wide-scale adoption of insecticide-treated nets (ITNs) has led to significant reductions in malaria morbidity and mortality worldwide. Delivery of ITNs to the 3.2 billion people at risk of malaria requires multiple steps in diverse settings. The effectiveness of the delivery of ITNs in order to prevent malaria relies on activities that include ITN manufacturing and design, integration into national and international malaria prevention policies, supplying and distributing ITNs to households and individuals, and, finally, programs focused on spurring demand for and use of ITNs by individuals at risk. This paper reviews some recent innovative strategies for ITN delivery across these four domains, places these innovations within the context of the history of ITN deployment, and identifies opportunities to further improve the effectiveness of this ubiquitous public health tool.
\end{abstract}

Keywords: malaria, insecticide-treated bed nets, prevention, access, ownership, use, policies

\section{Introduction}

In recent years, significant progress has been made in reducing malaria morbidity and mortality worldwide. ${ }^{1}$ In 2000, the Abuja Declaration and subsequent Millennium Development Goals mobilized billions of dollars of international resources to face an uncontrolled resurgence of malaria. Thanks to a significant investment in malaria control efforts from 2000 to 2015, clinical malaria cases in sub-Saharan Africa due to Plasmodium falciparum, the most common and severe form of malaria, were reduced by $40 \%$. Insecticide-treated nets (ITNs) have been recognized as the chief component of this progress, comprising an estimated $68 \%$ of cases averted due to their effectiveness and prevalence. ${ }^{2}$

Indeed, ITNs are well established as one of the most cost-effective forms of malaria prevention. Household use of an ITN leads to an 18\%-23\% reduction in child mortality. ${ }^{3,4}$ The World Health Organization (WHO) has called for universal access to ITNs, at a ratio of one net per two people (population ratio of 1:1.8), for all 3.2 billion people at risk of malaria worldwide. ${ }^{1}$ As a result, hundreds of millions of ITNs are distributed in malaria-endemic regions through various mechanisms every year.

Despite the general consensus that ITNs are a crucial component of malaria control strategies, there remain supply-side and demand-side barriers to meet the ambitious coverage goals set by the WHO. This review highlights innovative ITN delivery strategies that are being used to surmount some of these existing barriers to deliver these invaluable public health tools for every individual at risk of malaria. 


\section{Framework}

Effective delivery of ITNs as part of a successful malaria control strategy requires a variety of ITN-related activities encompassing the supply of and the demand for ITNs. ${ }^{5,6} \mathrm{~A}$ useful framework for conceptualizing this spectrum of activities is set out by Kilian et $\mathrm{al}^{7}$ in 2009 and subsequently adapted by Augustincic Polec et $\mathrm{al}^{6}$ in 2015. Figure 1 represents the author's own adapted framework. This framework provides four (occasionally overlapping) domains with a collection of related activities in which innovative approaches could help overcome obstacles to the ultimate goal of malaria prevention through the use of ITNs.

In this review, the supply-side activities include ITN-related policy choices (eg, targeting of particular populations or choosing a mix of public and private distribution venues) and physical activities related to transporting and distributing ITNs to individuals, and the demand-side activities include education and behavior change campaigns to promote "net cultures" in malaria susceptible regions, pricing schemes, and interventions to assist in effective use of ITNs. Policy decision about who should receive ITNs and how to ensure access to ITNs on a population basis impact both the supply-side and demandside. Finally, successful ITN delivery requires effective ITNs; therefore, there is an important role played by the "presupply" activities that involve developing novel insecticides, netting, and other innovations that improve ITNs themselves. The important concept here is that successful delivery of ITNs for malaria prevention involves both getting effective ITNs out to prospective users (supply-side) and ensuring that ITNs are used once they are available (demand-side).

\section{Methods}

In order to identify innovative ITN delivery strategies across the spectrum of ITN delivery, this review systematically explores the major domains set out in the aforementioned framework by searching for recent advances in both the peerreviewed and gray literature. The primary source of studies for this review was a (very general) search of PubMed and EMBASE for articles related to ITNs*, use*, ownership*, and malaria*. This database search involved screening of over 1,000 titles and abstracts published since 2010 looking for novel studies or approaches. The author subsequently performed Google searches in the areas of interest and handsearched through reference lists for promising studies and reports for additional studies of value. The gray literature (eg, web publications, reports, and news postings) is a rich source of cutting-edge activities in this area, as many innovative strategies arise from on-the-ground interventions by communitybased organizations or malaria-focused partnerships between local Ministries of Health and aid organizations (eg, United Kingdom-based Department for International Development, United States Agency for International Development [USAID], and Bill \& Melinda Gates Foundation), which may not have yet made it into the peer-reviewed literature.

For each domain, innovations are identified, summarized, and, where applicable, placed into the context of historical approaches to ITN delivery. Clearly, neither the proffered framework nor the list of included innovations is comprehensive; the author does not pretend to capture a universal map of ITN delivery activities or a list of all "cutting-edge" ITN delivery activities. This review is, rather, an attempt to highlight general thematic areas where innovative strategies are currently being employed and to call attention to outstanding areas of uncertainty and opportunity for future efforts. Finally, and importantly, the decision to include a study or report as "innovative" was based on a subjective synthesis of the literature and 13 years of experience with malaria in sub-Saharan Africa.

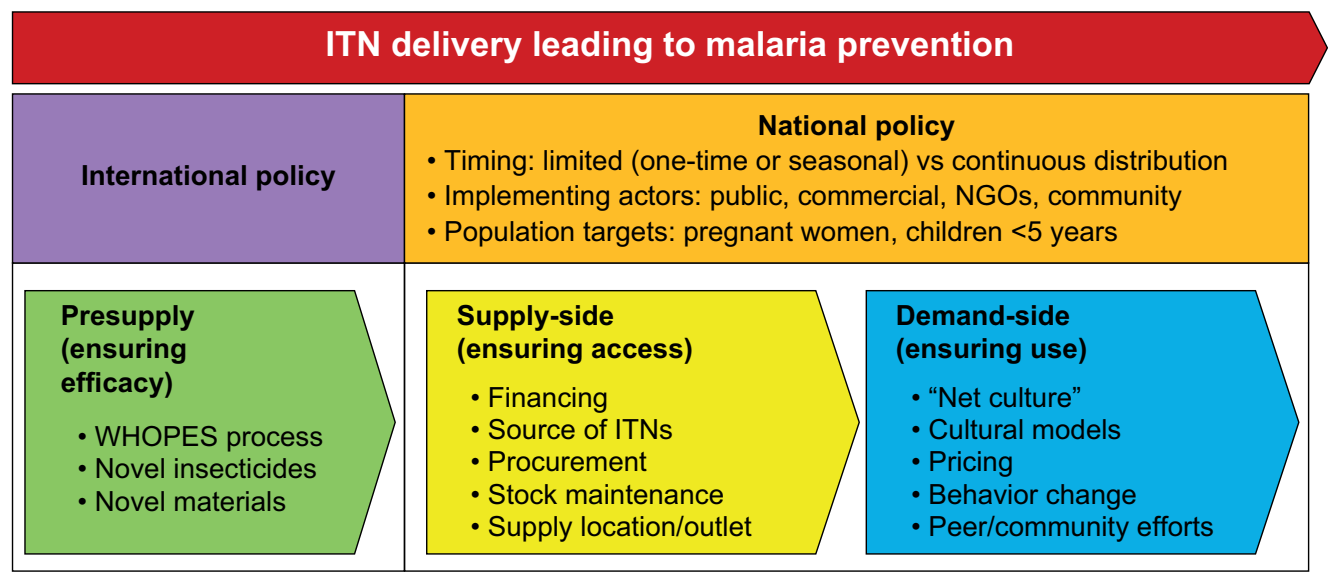

Figure I ITN delivery for malaria prevention.

Abbreviations: ITN, insecticide-treated net; NGOs, nongovernmental organizations; WHO, World Health Organization; WHOPES, WHO Pesticide Evaluation Scheme. 


\section{Presupply innovations in ITN delivery: ensuring effective ITNs}

As mentioned earlier, the success of ITNs as a malaria prevention tool is a direct function of their effectiveness in the field. The WHO defines an ITN as a net that "repels, disables, and/or kills mosquitoes coming into contact with insecticide on the netting material". ${ }^{8}$ While ITNs provide a barrier between malaria-infected mosquitoes and susceptible humans, the major mechanism of protection is through the insecticide component. The netting of the prototypical ITN is impregnated with insecticides that then provide a lethal dose to mosquitoes when they come into contact with the net. The insecticidal component of ITNs kills mosquitoes before they can infect individuals and reduces transmission between individuals in a community by eliminating the mosquito vector. ${ }^{9}$

One area ripe for innovation in this realm is the development of long-lasting insecticide nets (LLINs), which are subtypes of ITNs meant to last for longer time periods without requiring retreatment with insecticides. ${ }^{9}$ In the last decade, the WHO has made a concerted effort through recommendations via its WHO Pesticide Evaluation Scheme (WHOPES) mechanism to evaluate and promote the distribution of LLINs, which last for 3-5 years. ${ }^{10}$ Although there have been some studies that question the effectiveness of these ITNs over such long time periods, in terms of the long-term effectiveness of the insecticide delivery and in the practicality of whether ITNs can survive undamaged in households, LLINs are unquestionably the gold standard for ITNs. ${ }^{11}$ Evidence of innovation in this arena is the fact that WHOPES continues to review and recommend new commercial net products based on novel studies examining the safety and efficacy of new ITNs. ${ }^{12}$

Previously, only pyrethroid insecticides were approved for use in ITNs. ${ }^{8}$ An important area of recent innovation in the presupply domain is the development of novel insecticides for use in ITNs. This is a growing area of importance, as reports of pyrethroid-resistant mosquitoes are growing in Southeast Asia and sub-Saharan Africa ${ }^{13,14}$ as an extension of a long-recognized risk of vector evolution in response to widespread public health interventions. ${ }^{15}$ Although it is not clear that growing pyrethroid resistance is currently attenuating the effectiveness of ITNs, ${ }^{16}$ increased attention is being paid to adjunctive and alternative insecticides such as piperonylbutoxide and deltamethrin. ${ }^{17}$ Indeed, WHOPES has recently augmented its list of recommended LLINs to include multiple products using piperonylbutoxide and deltamethrin either in isolation or in combination with pyrethroids. ${ }^{9}$

Important barriers remain in developing the novel insecticides that are required to counteract resistance. The cost of developing novel insecticides could reach US\$200 million, making competing for these innovations to be less attractive to large commercial chemical companies. ${ }^{18}$ Recent innovative funding mechanisms are being employed by the Bill \& Melinda Gates Foundation and the Innovative Vector Control Consortium out of the Liverpool School of Tropical Medicine to spur commercial companies to explore their chemical libraries in the hope of identifying existing compounds that could be mobilized at cheaper costs for use in the public health domain. In addition to this concept of "active ingredient" screening of existing compounds, the Innovative Vector Control Consortium and others seek to identify and promote chemical engineering efforts focused on the molecular design of novel compounds for use as anti-mosquito insecticides. ${ }^{19}$

Another promising innovation in this domain is the use of new electrostatic coatings on the materials to increase the bioavailability of insecticides. ${ }^{20}$ In a recent study in Proceedings of the National Academy of Sciences of the United States of America, an electrostatic coating applied before insecticide impregnation led to increased mortality compared with traditional ITNs, even among mosquitoes that are resistant to the insecticide in the ITN. It is unclear how this method would perform in bed net applications, given the uncertainty of how the electrostatic polarity would hold up to frequent handling in daily use, but it demonstrates an example of innovation in material sciences as a new avenue for effectively controlling mosquito vectors.

\section{Policy innovations in ITN delivery: planning ITN programs}

As discussed, the WHO currently recommends that all individuals in malaria-endemic settings have access to ITNs at night for protection against malaria. Before proceeding to delivering ITNs for individual access, countries at risk of malaria must define policies for how ITNs need to be distributed. In this section, the author tries to highlight the higher-level policy decisions that have a general impact on both ensuring access to (supply-side) and ensuring use of (demand-side) ITNs and discusses policy decisions that are unique to those domains in the subsequent sections.

A significant change affecting individual countries' malaria policy programs came with the launch of the President's Malaria Initiative (PMI) in 2005. PMI, which is housed within the USAID and implemented with the Centers for Disease Control, represents a significant effort to coordinate malaria strategy across the US government. PMI produces global malaria prevention strategy documents, covering 2009-2014 and now 2015-2020, ${ }^{21}$ which closely mirror the 
$\mathrm{WHO}^{22}$ and Roll Back Malaria Partnership ${ }^{23}$ global strategy documents.

In addition, PMI works with partner countries to develop individualized annual Malaria Operational Plans (MOPs) that cover all aspects of malaria control and treatment. ${ }^{24}$ As pertains to ITNs, these individualized operational plans establish important policy choices by national malaria prevention programs and provide a common understanding of ITN policies between national governments and bilateral donors, such as USAID/PMI. For effective implementation of prevention goals, nationwide policies are useful in harmonizing supply-side and demand-side considerations. Explicit policy decisions must be made with regard to, among many others, which entities, governmental or nongovernmental, are charged with implementing ITN delivery strategies, the methods for distributing ITNs, who will promote ITN use, how ITNs will be promoted in at-risk communities, and how to coordinate wide-scale campaigns for ITN distribution.

Although the formal policy setting exercise represented by annual USAID/PMI Malaria Operational Plans appears to be effective in coordinating improvements in malaria control, ${ }^{25}$ there is also evidence that there can be advantages in flexibility in policy decisions. There is an interesting recent study of anti-larviciding policy in Nigeria ${ }^{26}$ that, while not specific to ITN policy, suggests lessons applicable to other aspects of malaria control programs such as ITNs. This study discusses policy changes that were made outside of the normative policy process in order to adapt to current needs and opportunities. In this case, an extraordinary effort was made to implement larvicide interventions even though such interventions were outside the classic malaria control policy framework. Some of the lessons from this case study were as follows: 1) the value of high-level stakeholders who have the bureaucratic power to chart paths outside of normative process and 2) the explicit connection between malaria control objectives and government concerns external to the typical public health arena (eg, economic development). Therefore, although national and bilateral coordination through formal policy setting appears valuable, there may be a role for ITN policy innovations that work outside the existing frameworks in situations that represent a need for an urgent response (eg, malaria epidemics), changing conditions (eg, rise of insecticide resistance), or new opportunities (eg, new and more effective type of ITN available).

Important policy considerations include, among others, the choice of which populations to target with ITN distribution programs, which organizations will implement the programs, and whether programs will be continuous or consist of large-scale campaigns that are discrete in time. The general consensus is that countries should use a wide variety of mechanisms to tailor their malaria control programs to that country's particular mix of malaria risks and population health concerns. ${ }^{19-21}$ The current understanding is that each policy decision must be made in a coordinated manner that takes into account potential unintended consequences due to the interwoven nature of malaria control programs. Policy decisions to use particular actors for malaria program implementation or to target particular groups for coverage run the risk of crowding out other aspects of national programs. Plans to use targeted subsidies to increase ITN access for women, for example, have to be weighed against the potential for a loss of legitimacy and engagement with men in preventing malaria. ${ }^{27}$ As discussed earlier, the most successful malaria control policy regimes are the ones that involve multiple stakeholders who are required to implement, and are impacted by, ITN delivery policies.

\section{Supply-side innovations in ITN delivery: ensuring ITN access}

Ensuring that households and individuals have access to ITNs is the next crucial component for effective ITN delivery. From a programmatic level, this involves multiple steps required to maintain a ready supply of ITNs: 1) at various outlets (where) and 2) according to the schedule chosen by national policy-makers (when).

\section{Financing}

Most typically, national programs identify the funds for ITNs by working with donors comprised of governmental (eg, USAID and Department for International Development) or nongovernmental (eg, Gates Foundation) actors or combinations of the two (government-nongovernmental organization partnerships). These normative and deliberative mechanisms for funding are less well suited to covering newly identified gaps in ITN policy or adjusting to rapidly arising needs, as in an epidemic setting.

In 2011, an impending malaria epidemic in Zambia spurred the first use of the Pledge Guarantee for Health to streamline the process for obtaining 800,000 ITNs for delivery to Zambian households at a risk of malaria. ${ }^{28}$ This process involves ad hoc creation of partnerships to address urgent funding and procurement needs. In the Zambia case, the United Nations Foundation brokered a deal between a private bank and the World Bank to guarantee a loan to cover the cost of the ITNs through the UN Children's and Education Fund. The entire financing and procurement process took only 
2 months, in contrast to the 5-6 months typically required. Such innovative financing mechanisms can overcome the barriers to the efficient procurement of ITNs for delivery to at-risk populations.

\section{Source}

Another consideration in obtaining ITNs is the source from which they are procured. With the development of a market within Africa for quality ITNs, local companies have been developed which can serve local demand for ITNs and act as a foundation for local capacity building and technology development through so-called south-south collaborations. ${ }^{29}$ An example of innovation in local ITN manufacturing is A to $\mathrm{Z}$ textiles, a company based in Tanzania. ${ }^{30} \mathrm{~A}$ to $\mathrm{Z}$ creates high-quality ITNs that are locally manufactured in Tanzania and are the only African-produced ITNs with WHOPES certification. The technological capacity to produce ITNs within Africa creates a road map for alternative means of streamlining the procurement process leveraging the local manufacturing of ITNs.

\section{Outlet}

The location of delivery of ITNs ranges widely and should likely involve a diverse mix of outlets in order to ensure wide access to ITNs depending on the malaria-risk profile. ${ }^{8}$ Potential channels for ITN supply can range from commercial retailers to clinic visits to delivery of ITNs directly to households or individuals in the community. Although WHO recommends universal coverage, many countries in policy or practice continue to target primarily pregnant women and children $<5$ years of age. ${ }^{1}$ Since free distribution of ITNs is capital intensive, such campaigns are typically heavily reliant on donor funds. Because donor funds can be episodic, the commercial sector can provide a valuable continual distribution mechanism through which at-risk individuals and households can obtain ITNs, even if they are not a part of the targeted populations or there are interruptions in the mass distribution campaigns. In addition, the continuous availability of ITNs at commercial suppliers contributes to a "net culture" that helps to buoy demand for ITNs overall and facilitate delivery" (discussed more in "Demand-side innovations in ITN delivery: ensuring ITN use" section).

Innovative partnerships between governments, donors, and the commercial sector can lead to the provision of ITNs that supplement coexistent government mechanisms. In Uganda, for example, through the AFFORD project, ${ }^{31}$ commercial suppliers received price support for the sale of higher-quality, WHOPES-approved ITNs to allow for their sale at the same price as that of low-quality untreated bed nets. Retailers also received funding support for productspecific marketing, after they had committed some of their own funds. These deliberate price and marketing supports aided in shaping the market for high-quality ITNs. These activities were a component of a successful increase in access to ITNs, from 80,000 bed nets in 1999 to 3 million in 2004. Although there were some issues, such as the full price subsidies not always being passed on to the consumer, public-private partnerships of this kind represent innovative means of ensuring that households have continual access to high-quality ITNs outside of dependence on the typical episodic donor funding cycle.

Another important outlet for the supply of ITNs is in the community. A study in Niger compared the communitybased supply of ITNs versus routine, facility-based, and found higher ITN ownership, more equitable coverage, and reduced ITN leakage in the community-based program. ${ }^{32}$ A systematic review and meta-analysis of malaria control programs found that community-based interventions, including ITN supply, were as effective as facility-based care, with suggestive evidence that community interventions result in better malaria-relevant outcomes, such as reduced malaria prevalence, anemia, and mortality. ${ }^{33}$

Community-based ITN supply programs, especially when the interventions are implemented by community health workers (CHWs), leverage local knowledge and the prestige and influencing power of local agents. Population Services International and others have championed a model of "social marketing" which uses marketing concepts to address public health concerns. In Madagascar, for example, Population Services International supplies subsidized ITNs to trained local CHWs, who then resell the ITNs in their communities at a small profit. ${ }^{34}$ These innovative mechanisms seek to combine the power of markets to match supply and demand with the community-based expertise of local actors. Even when community programs are not directly supplying the ITNs, they can play important roles in ensuring ITN access through advocating for their community members, registering households/ individuals prior to ITN campaigns, providing surveillance data about ITN ownership and use pre- and post-distribution campaigns, and ensuring equitable coverage of ITNs. ${ }^{35}$

\section{Stock management}

Once the preferred outlet mechanism for ITNs is chosen, it is important to maintain an adequate supply at those outlets. Stock-outs of ITNs are a common cause of the failure of ITN distribution mechanisms, especially those meant to provide 
a continuous supply (eg, provision of ITNs at antenatal visits for pregnant women). ${ }^{36}$ Various innovative processes have been developed to face the stock-out challenge that is prevalent in poorly funded health systems.

A comparison of the costs and efficiency of various "last mile" distribution systems by USAID in Nigeria suggests that some methods of delivery and information capture outperform others. ${ }^{37}$ In a comparison of four methods of ITN supply, USAID found that information gathering performed at the time of ITN delivery by nonservice delivery personnel (ie, those without clinic responsibilities) was the least costly and most efficient. This method outperformed other methods of maintaining existing stock supplies that might rely on busy clinic staff to also maintain stock counts or regular inventory counts. Evaluations such as this can provide best-of-practice information for Nigeria and for other countries in designing their ITN supply systems.

Mobile phone technologies have been used to improve stock management efficiency. A study in Uganda suggests that the use of short message service (SMS) systems reduces stock-out rates of ITNs and rapid diagnostic tests (RDTs). ${ }^{38}$ Nigeria has also used SMS systems to facilitate the distribution of ITNs along the entire process: from central stocks to holding warehouses to retailers, and eventually the CHWs report their delivery directly to households in at-risk communities. ${ }^{39}$ The messages are sent using special "shortcodes" that allow them to be put through free of charge and are donated by the local telecoms in partnership with the UN Children's and Education Fund.

The USAID Deliver Project has published additional guidance on supply chain planning and management for ITNs. ${ }^{40}$ These suggestions take inspiration from lessons learned from commercial supply chain management and offer the opportunity for the use of modeling software to analyze how supply chain management decisions interact. Activities include basic supply chain system design and both network and transportation optimization. Supply-side challenges are often predictable through an understanding of a country's prior experience or borrowing of lessons from other countries with similar environments. Much as in the policy arena, deliberate planning for how to tackle these challenges can lead to innovative means of resolving them and should include liberal borrowing of best practices from other locales.

Another interesting publication from USAID Deliver Project explores the potential for supply chain mechanisms that work outside the existing central medical stores model. ${ }^{41}$ It argues that supply chains, especially for vital commodities such as ITNs, must be flexible and adaptable to local require- ments. This may involve, in some cases, a de-emphasis on the traditional central medical stores, which is a centralized model, to involve facilities having direct access to suppliers, privatization of some central medical stores roles, and creation of competition between agencies responsible for ITN supplies to incentivize efficiency and performance. Although these mechanisms may challenge classical hierarchical supply regimes, and thus may face barriers to adoption, they demonstrate that there remains room for open thinking and innovative application of organizing principles and incentives in order to improve the performance of ITN supply systems and decrease stock-outs that interfere with ensuring ITN access for those at risk of malaria.

\section{Demand-side innovations in ITN delivery: ensuring ITN use}

Although the effectiveness of ITN materials; smart policies for their financing, procurement, and distribution, and reliable supply chains are all necessary for the realization of the potential for ITNs to prevent malaria, a crucial step in this cascade is the consistent and effective use of ITNs by individuals at risk of malaria. Without dependable and predictable use of ITNs by at-risk individuals, the millions of dollars invested in ITNs are, at best, inefficient or, at worst, misguided.

Demand for ITNs must be stimulated in a vast diversity of environments. At its most disaggregated, demand for and use of ITNs may be a unique characteristic at even the individual level. While a complete discussion of the determinants of individual use of ITNs is beyond the scope of this review, the diversity of the prospective audience makes ensuring the use of ITNs one of the most vibrant areas for innovation in delivering ITNs for malaria prevention (see Pulford et $\mathrm{al}^{42}$ and Singh et $\mathrm{al}^{43}$ ).

A "net culture", sustained demand for and use of ITNs, has been identified as a critical component of a healthy malaria prevention program. ${ }^{6}$ Creating a net culture involves general malaria education, introduction and exposure to ITNs, and the placement of ITNs into a cultural milieu that is accessible to those at risk of malaria. PMI's Malaria Communities Program, for example, seeks to innovate around creating social pressures and altering social norms to promote ITN use. Such activities must be different depending no the community and cultural context: village chiefs in Malawi were asked to speak out against the use of nets for fishing, whereas in Mozambique ITN programs confronted a lack of prioritization of women and children due to a cultural bias toward ITN use by the men in the household. ${ }^{44}$

Another approach tailored to the local cultural context has been used in Ethiopia where schoolchildren receive green, 
yellow or red cards designating whether their household uses bed nets. The CHWs also mark the outside of the homes with the color codes, ensuring consistent pressures in favor of ITN use from both within and outside the household..$^{45}$

The BOMA model in Kenya is an example of innovative health education and provision. This program, among seminomadic Maasai peoples, focuses directly on accommodating cultural barriers to understanding of Western illness concepts. ${ }^{46}$ Although such explicit cultural barriers may be less apparent in more Westernized African contexts, it is likely that ITN education and promotion campaigns that consider local cultural contexts will be more effective in stimulating a net culture than those which do not.

Behavior change campaigns are a traditional method for attempting to boost demand for ITNs. Innovations are allowing these campaigns to evaluate their effectiveness and access new populations. A study in Cameroon, for example, demonstrated that the KO Palu campaign, which used television, radio, community theater, SMS, and celebrity endorsements to increase awareness of malaria and ITNs, led to a significant increase in household use of ITNs. ${ }^{47}$ Other mobile technologies are increasingly being used to improve ITN use. TTC Mobile and various nongovernmental organization partners in Mali are using an SMS-based malaria quiz, with prizes to incentivize participation, to promote malaria awareness and to gather data about ITN use among individuals. ${ }^{48}$

Access to affordable ITNs is a crucial component of their delivery in impoverished households who may not be able to afford them. Free delivery, either in mass community campaigns or in targeted maternal or pediatric clinic visits, is one mechanism for their delivery, but many households have a need for ITNs outside these mechanisms either to cover other household members or when free delivery is not available (eg, stock-outs). Various programs for ITN subsidization have been used to enhance their adoption. These programs typically provide vouchers to targeted individuals and households in exchange for subsidized ITNs at distributors. ${ }^{49}$ Voucher systems, such as the well-known Tanzania National Voucher Scheme, have been found to be cost-effective. ${ }^{50}$

A similar program in Ghana uses an e-voucher system whereby individuals are provided a paper coupon with a special code entitling them to a subsidized ITN. On redeeming the coupon at a retail outlet, the retailer submits the code using a mobile phone to be reimbursed for the difference between the retailer's costs and the subsidy. This system allows for continuous monitoring of coupon redemption via an online database and reduces fraud by ensuring that the code is used only once. ${ }^{51}$ Tanzania has also developed an e-voucher/mobile phone system to make their voucher scheme more efficient and found a $7.5 \%$ cost reduction compared to the traditional scheme. ${ }^{52}$

Efforts to boost demand for ITNs and incentivize their use are areas ripe for innovation. A randomized controlled trial in Madagascar in 2007 demonstrated that providing small "prizes" (household goods) in exchange for household ITN use (assessed by visual observation) led to a 27\% increase relative to households who did not receive incentives after 1 month. Although differences in ITN use between the two groups disappeared after 6 months with the removal of the incentive, this study demonstrated a means of significantly boosting ITN use in at-risk households with only small incentives. ${ }^{53}$

Another area of innovation for improving ITN adherence involves novel tools for measuring ITN use. The Consortium for Affordable Medical Technologies, affiliated with the Massachusetts General Hospital Center for Global Health, maintains an innovation laboratory in Uganda affiliated with the Mbarara University of Science and Technology. The Consortium for Affordable Medical Technologies uses the concept of co-creation, which engages end users as partners throughout product development. ${ }^{54}$ By engaging local students, engineers, and end users in Uganda, the Consortium for Affordable Medical Technologies is spearheading a variety of ITN-related innovations, including a novel electronic adherence monitor for ITN use called SmartNet and a flexible frame concept called FlexNet for facilitating the use of ITNs in locations outside the home. Combining end-user input with local manufacturing allows for rapid incorporation of design modifications and increases the likelihood that these technology innovations are successful in the prototype-tofield technology transition. SmartNet and other novel tools for researching ITN adherence ${ }^{55}$ represent valuable innovations in understanding the effectiveness of ITN delivery at its terminal event: actual use by at-risk individuals. Objective adherence monitoring tools such as SmartNet allow for a more accurate and precise measurement of ITN use than what is afforded by self-reports, the current standard.

\section{Conclusion}

ITNs are a ubiquitous health technology that is used in many regions of the world susceptible to malaria infection. Across the continuum of activities required to effectively deliver ITNs to those in need of malaria prevention there, there are many and diverse opportunities for innovation. This review has provided an overview of some of these innovations with the hope of spurring continuing experimentation with novel 
strategies for improving the ways that ITNs can best be designed, distributed, and promoted to prevent malaria for the billions of people at risk throughout the world.

\section{Disclosure}

The author reports no conflicts of interest in this work.

\section{References}

1. World Health Organization. World Malaria Report. Geneva: World Health Organization; 2015. Available from: http://apps.who.int/iris/bitstr eam/10665/200018/1/9789241565158_eng.pdf. Accessed March 4, 2016.

2. Bhatt S, Weiss DJ, Cameron E, et al. The effect of malaria control on Plasmodium falciparum in Africa between 2000 and 2015. Nature. 20158;526(7572):207-211.

3. Lengeler $C$. Insecticide-treated bed nets and curtains for preventing malaria. Cochrane Database Sys Rev. 2004;(2):CD000363.

4. Lim SS, Fullman N, Stokes A, Ravishankar N, Masiye F, Murray CJ, Gakidou E. Net benefits: a multicountry analysis of observational data examining associations between insecticide-treated mosquito nets and health outcomes. PLoS Med. 2011;8(9):e1001091.

5. Sexton R. Best practices for an insecticide-treated bed net distribution programme in sub-Saharan eastern Africa. Mal J. 2011;10:157.

6. Augustincic Polec L, Petkovic J, Welch V, et al. Strategies to increase the ownership and use of insecticide-treated bednets to prevent malaria: a systematic review. Cochrane Database Syst Rev. 2015:30(3):CD009186.

7. Kilian, A, Wijayanandana, N, Ssekitoleeko, J. Review of delivery strategies for insecticide treated mosquito nets: are we ready for the next phase of malaria control efforts? TropIKA.net. 2010;1(1).

8. World Health Organization. Global Malaria Programme, InsecticideTreated Mosquito Nets: A WHO Position Statement. Geneva: World Health Organization; 2007. Available from: http://can-mnch.ca/wpcontent/uploads/2013/11/Insecticide-Treated-Mosquito-Nets-a-WHOPosition-Statement1.pdf. Accessed August 25, 2016.

9. CDC. Insecticide-treated bed nets. Available from: http://www.cdc.gov/ malaria/malaria_worldwide/reduction/itn.html. Accessed March 6, 2016.

10. World Health Organization. WHO Recommended Long-Lasting Insecticidal Nets. Geneva: WHO; 2015. Available from: http://www.who. int/entity/whopes/Long-lasting_insecticidal_nets_November_2015. pdf. Accessed February 2, 2016.

11. Pulkki-Brännström A, Wolff C, Brännström N, Worrall J. Cost and cost effectiveness of long-lasting insecticide-treated bed nets-a model-based analysis. Cost Eff Resour Alloc. 2012;10:5.

12. World Health Organization. Report of the Eighteenth WHOPES Working Group Meeting - Review of MiraNet LN, Panda Net 2.0 LN, Yahe LN and SafeNet LN. Geneva: World Health Organization Headquarters; 29 June to 1 July 2015. Available from: http://apps.who.int/iris/bitstr eam/10665/184034/1/9789241509428_eng.pdf. Accessed February 3, 2016.

13. Asidi A, N'Guessan R, Akogbeto M, Curtis C, Rowland M. Loss of household protection from use of insecticide-treated nets against pyrethroid-resistant mosquitoes, Benin. Emerg Infect Dis. 2012;18(7).

14. Chanda E, Hemingway J, Kleinschmidt I, et al. Insecticide resistance and the future of malaria control in Zambia. PLoS One. 2011;6(9):e24336.

15. Nauen R. Insecticide resistance in disease vectors of public health importance. Pest Manag Sci. 2007;63(7):628-633.

16. Strode C, Donegan S, Garner P, Enayati AA, Hemingway J. The Impact of pyrethroid resistance on the efficacy of insecticide-treated bed nets against African Anopheline mosquitoes: systematic review and metaanalysis. PLoS Med. 2014;11(3):e1001619.

17. Yewhalaw D, Asale A, Tushune K, Getachew Y, Duchateau L, Speybroeck N. Bio-efficacy of selected long-lasting insecticidal nets against pyrethroid resistant Anopheles arabiensis from South-Western Ethiopia. Parasit Vectors. 2012;5:159.
18. Insecticide Resistance Action Committee (IRAC). Manual: Prevention and Management of Insecticide Resistance in Vectors of Public Health Importance. 2nd ed.; 2010

19. McLean T. IVCC Strategic Plan 2006-2011. The Current Status of Vector Control $R \& D$. Available from: http://www.rollbackmalaria.org/ files/files/partnership/wg/wg_procurementsupply/docs/7psmwgTom McLean_IVCC.pdf. Accessed February 3, 2016.

20. Andriessen R, Snetselaar J, Suer R, Osinga A, Deschietere J, et al. Electrostatic coating enhances bioavailability of insecticides and breaks pyrethroid resistance in mosquitoes. PNAS. 2015;112(39):12081-12086.

21. Malaria Eradication Scientific Alliance. President's Malaria Initiative Malaria Strategy 2015 - 2020. Barcelona: MESA; 2015. Available from: http://www.malariaeradication.org/knowledge-hub/presidents-malariainitiative-malaria-strategy-2015-2020. Accessed August 25, 2016.

22. World Health Organization. Global Technical Strategy for Malaria 2016-2030; 2015. Available from: http://www.who.int/malaria/publications/atoz/9789241564991/en/. Accessed March 4, 2016.

23. World Health Organization Secretariat, on behalf of the Roll Back Malaria Programme. Action and Investment to Defeat Malaria 2016-2030. For a Malaria-Free World; 2014. Available from: http://www.rollbackmalaria. org/about/about-rbm/aim-2016-2030. Accessed March 4, 2016.

24. FY 2016. Malaria Operational Plans; 2016. Available from: http:// www.pmi.gov/resource-library/mops. Accessed March 4, 2016.

25. Simon J, Yeboah-Antwi K, Schapira A, et al. External evaluation of the President's Malaria Initiative. Final Report. Washington, DC, Global Health Technical Assistance Project [218] p. (Report No. 11-01-545); 2011. Available from: http://www.pmi.gov/docs/default-source/default-documentlibrary/pmi-reports/audit_fullreport.pdf. Accessed March 4, 2016.

26. Tesfazghi K, Hill J, Jones C, Ranson H, Worrall E. National malaria vector control policy: an analysis of the decision to scale-up larviciding in Nigeria. Health Policy Plan. 2016;31(1):91-101.

27. World Health Organization (WHO). Targeted Subsidy Strategies for National Scaling up of Insecticide-Treated Netting Programmes Principles and Approaches. Geneva: WHO; 2005.

28. Mungcal I. New Financing Mechanism Cuts Time between Grant Approval, Aid Delivery; 2011. Available from: https://www.devex.com/ news/how-anti-malaria-bednets-were-delivered-to-zambia-ahead-ofschedule-76671. Accessed March 7, 2016.

29. Shah R, Singer P, Daar A. Science-based health innovation in Tanzania: bednets and a base for invention. BMC Intern Health Human Right. 2010;10(Suppl 1):S4.

30. Masum H, Shah R, Schroeder K, Daar A, Singer P. Africa's largest longlasting insecticide-treated net producer: lessons from A to Z Textiles. BMC Intern Health Human Right. 2010;10(Suppl 1):S6.

31. Kilian, A. Insecticide treated nets: balancing the roles of the commercial and public sectors. Case study of three countries in sub-Sahara Africa, Malaria Consortium Learning Paper Series; 2013. Available from: www. malariaconsortium.org/pages/learning-papers.htm. Accessed March 7, 2016.

32. Nonaka D, Maazou A, Yamagata S, et al. Distribution of subsidized insecticide-treated bed nets through a community health committee in Boboye Health District, Niger. Trop Med and Health. 2012;40(4): 125-131.

33. Salam R, Das J, Lassi Z, Bhutta Z. Impact of community-based interventions for the prevention and control of malaria on intervention coverage and health outcomes for the prevention and control of malaria. Infect Dis Poverty. 2014;3:25.

34. Advancing Partners \& Communities. Country Profile: Madagascar Community Health Programs. Arlington, VA: Advancing Partners \& Communities; 2013.

35. USAID. Achieving, Tracking, and Maintaining High ITN Coverage: Community Strategies. Malaria Communities Program; 2013. Available from: http://www.mchip.net/sites/default/files/MCP\%20ITN\%20 Case\%20Study.pdf. Accessed March 1, 2016.

36. Theiss-Nyland K, Ejersa W, Karema C, et al. Operational challenges to continuous LLIN distribution: a qualitative rapid assessment in four countries. Malar J. 2016;15(1):131. 
37. USAID | Deliver Project. Evaluating Last Mile Distribution Systems in Nigeria; 2014. Available from: http://deliver.jsi.com/dlvr_content/resources/ allpubs/countryreports/NG_EvalLastMile.pdf. Accessed March 6, 2016.

38. Asiimwe C, Gelvin D, Lee E, et al. Use of an innovative, affordable, and open-source short message service-based tool to monitor malaria in remote areas of Uganda. Am J Trop Med Hyg. 2011;85(1): 26-33.

39. UNICEF. Text Messages Bolster World's Largest Distribution of Mosquito Nets. Available from: http://www.unicef.org/health/nigeria_53421. html. Accessed March 6, 2016.

40. USAID | Deliver Project. Optimizing Supply Chains for Improved Performance; 2014. Arlington, VA: USAID | Deliver Project, Task Order 4. Available from: http://deliver.jsi.com/dlvr_content/resources/allpubs/ logisticsbriefs/OptiSuppChai.pdf. Accessed March 6, 2016.

41. USAID | DELIVER PROJECT. Exploring Supply Chain Augmentation for Malaria Commodities; 2012. Arlington, VA: USAID | Deliver Project, Task Order 7. Available from: http://deliver.jsi.com/dlvr_content/ resources/allpubs/logisticsbriefs/ExplSCAugmMala.pdf. Accessed March 6, 2016.

42. Pulford J, Hetzel M, Bryant M, Siba P, Mueller I. Reported reasons for not using a mosquito net when one is available: a review of the published literature. Malaria J. 2011;10:83.

43. Singh M, Brown G, Rogerson S. Ownership and use of insecticidetreated nets during pregnancy in sub-Saharan Africa: a review. Malar J. 2013;12:268.

44. PMI. Malaria Communities Program. Achieving, Tracking, and Maintaining High ITN Coverage: Community Strategies; 2013. Available from: http://www.mchip.net/sites/default/files/MCP\%20ITN\%20 Case\%20Study.pdf. Accessed May 3, 2016.

45. PMI. Household Incentives for Bed Nets; 2015. Available from: https:// pmi.gov/news/stories-from-the-field/stories-from-the-field---detail/ household-incentives-for-bed-nets. Accessed March 7, 2016.
46. The African Medical and Research Foundation (AMREF). The 'BOMA' Health Delivery Model: An Innovative Approach to Delivering Maternal, Newborn and Child Health Services to Semi-Nomadic Communities in Hard-To-Reach Regions; 2013. Available from: http://amref.org/ download/521362a0821a0/. Accessed March 6, 2016.

47. Bowen H. Impact of a mass media campaign on bed net use in Cameroon. Malar J. 2013;12:36.

48. TTC Mobile. Using the power of information to prevent malaria. Available from: http://ttcmobile.com/the-power-of-information-to-preventmalaria/. Accessed May 3, 2016.

49. Worrall E, Hill J, Webster J, Mortimer J. Experience of targeting subsidies on insecticide-treated nets: what do we know and what are the knowledge gaps? Trop Med Int Health. 2005;10(1):19-31.

50. Mulligan JA, Yukich J, Hanson K. Costs and effects of the Tanzanian national voucher scheme for insecticide-treated nets. Malar $J$. 2008; 7:32.

51. Tropical Health LLP. Ghana LLIN e-Coupon Pilot Process Evaluation. Available from: https:/www.k4health.org/sites/default/files/ecoupon_process_evaluation_final_report.pdf. Accessed March 7, 2016.

52. Networks, USAID, RBM. Making Targeted Subsidies Fast and Flexible-The TNVS eVoucher. Available from: http://www.rollbackmalaria. org/files/files/resources/MalariaLIB7Tanzania.pdf. Accessed March 6, 2016.

53. Krezanoski P, Comfort A, Hamer D. Effect of incentives on insecticidetreated bed net use in sub-Saharan Africa: a cluster randomized trial in Madagascar. Malar J. 2010;9:186.

54. Caldwell A, Young A, Gomez-Marquez J, Olson KR. Global Health Technology 2.0: leaping over the gap with collaborative invention. IEEE Pulse. 2011;2(4):63-67.

55. Koudou BG, Malone D, Hemingway J. The use of motion detectors to estimate net usage by householders, in relation to mosquito density in central Cote d'Ivoire: preliminary results. Parasit Vectors. 2014;7:96.
Research and Reports in Tropical Medicine

\section{Publish your work in this journal}

Research and Reports in Tropical Medicine is an international, peerreviewed, open access journal publishing original research, case reports, editorials, reviews and commentaries on all areas of tropical medicine, including: Diseases and medicine in tropical regions; Entomology; Epidemiology; Health economics issues; Infectious disease; Laboratory

\section{Dovepress}

science and new technology in tropical medicine; Parasitology; Public health medicine/health care policy in tropical regions; and Microbiology. The manuscript management system is completely online and includes a very quick and fair peer-review system. Visit http://www.dovepress. com/testimonials.php to read real quotes from published authors. 